\title{
Interval estimation of voltage magnitude in radial distribution feeder with minimal data
} acquisition requirements

\section{Rasmussen, Theis Bo; Yang, Guangya; Nielsen, Arne Hejde}

Published in:

International Journal of Electrical Power \& Energy Systems

Link to article, DOI:

10.1016/j.ijepes.2019.05.041

Publication date:

2019

Document Version

Peer reviewed version

Link back to DTU Orbit

Citation (APA):

Rasmussen, T. B., Yang, G., \& Nielsen, A. H. (2019). Interval estimation of voltage magnitude in radial distribution feeder with minimal data acquisition requirements. International Journal of Electrical Power \& Energy Systems, 113, 281-287. https://doi.org/10.1016/j.ijepes.2019.05.041

\section{General rights}

Copyright and moral rights for the publications made accessible in the public portal are retained by the authors and/or other copyright owners and it is a condition of accessing publications that users recognise and abide by the legal requirements associated with these rights.

- Users may download and print one copy of any publication from the public portal for the purpose of private study or research.

- You may not further distribute the material or use it for any profit-making activity or commercial gain

- You may freely distribute the URL identifying the publication in the public portal 


\title{
Interval estimation of voltage magnitude in radial distribution feeder with minimal data acquisition requirements
}

\author{
Theis Bo Rasmussen, Guangya Yang, Arne Hejde Nielsen \\ Department of Electrical Engineering, Technical University of Denmark, 2800 Kgs. Lyngby, Denmark
}

\section{ART ICLE INFO}

\section{Keywords:}

Cyber-physical power system

Interval estimation

Voltage monitoring

Smart meters

Data privacy

\begin{abstract}
A B S T R A C T
The increased complexity of electric distribution networks, entails higher requirements on the interoperability between the information and communication technology (ICT) infrastructure and the power system. In current literature, distribution network monitoring methods require reliable operation of the ICT infrastructure, and depend on load estimation accuracy in cases of unavailable measurements. In the low voltage (LV) distribution network, ICT infrastructure is sensible to noise and congestion, and load estimation is challenged by irrational consumer behaviour. Such limitation impede the implementation of currently proposed methods. The conditions are complicated further when considering the impact of emerging data privacy concerns that can prevent utilization of household consumption data.

Therefore, this work proposes a novel method for voltage magnitude interval estimation by utilizing the in formation from existing data acquisition systems in LV distribution networks. The proposed method is based on knowledge of load connection, and estimates the entire radial feeder voltage magnitude conditions from measurements of voltage magnitudes from minimum one node. The proposed method can therefore be implemented on existing ICT infrastructure and effectively keeps the privacy of consumers consumption profiles. The ability of the proposed method to estimate the feeder voltage interval is illustrated by an example and evaluated using Monte Carlo simulations for different meter distribution and accuracy scenarios.
\end{abstract}

\section{Introduction}

In recent years, the information and communication technology (ICT) infrastructure connected to the power system has seen a gradual upgrade through digitalization of substations and deployment of smart meters $[1,2]$. While these infrastructure changes improve measurement availability, the size, diversity and distribution of the power system complicates network monitoring with increased requirements on data communication, processing and analysis [3].

The investment in ICT infrastructure is entailed by the electrification of services and ongoing implementation of distributed generation, which has increased the complexity of distribution networks. This change is especially true at the low voltage (LV) level, due to high R/X ratios, unbalanced phases, and uncertainties regarding grid interactions at individual load points. Such networks require improved operational assessment to accommodate higher levels of renewable integration and activation of smart grid strategies, that can support the grid through active participation of distributed energy resources (DERs), e.g. photovoltaics (PV) and electric vehicles (EVs).

The challenge in both the physical and cyber system aspects of a modern power system has entailed a transition of the traditional power system into a cyber-physical system [4]. In this context, applications are being developed to exploit the information harvested by the ICT infrastructure, providing an overview of network operation. On the distribution grid side, there exists different approaches to estimate the operation. The simplest method is through direct measurements of secondary transformer loading. While this simple approach historically has been applicable in traditional distribution networks, a large degree of uncertainty is expected in future distribution networks due to the electrification of residential services and intermittent nature of renewables [5]. As a consequence, different methods propose to implement additional measurement points within distribution networks $[6,7]$.

Using a higher spatial resolution of meters within the distribution network enables observation of network conditions through acquiring consumption measurements, and calculating the power flow through methods designed for radial feeders, such as the DistFlow branch flow method [8-10]. Since the distributed sensors have imperfect accuracy, different computational approaches to uncertainty modeling in power flow exists, including probabilistic and interval arithmetic based methods [11]. Such methods give an estimation of the grid conditions, and therefore provide a useful indication of the operational conditions [12].

For a more detailed assessment of network operation, distribution system state estimation (DSSE) methods are proposed. In comparison to state estimators at transmission level, DSSEs are more complicated 
due to the physical characteristics and the inferior performance and reliability of the cyber system in distribution networks $[13,14]$. Current research in DSSE has moved from considering different state variables and state equation representations [15-17], towards utilization of the increased meter distribution that are based on an assumption of time synchronized measurement acquisition $[18,19]$. But while transmission networks metering assests are connected through mainly optical fibres, distribution network communication infrastructure is less reliable and more vulnerable to noise and congestion $[20,21]$. Therefore, the implementation of DSSE is heavily impeded by the ICT infrastructure, which has been revealed in different demonstration studies [22,23].

To deal with the incomplete datasets from unreliable ICT infrastructure, pseudo-measurement generation methods are proposed to give an idea of the load point interactions and thereby estimate/model load point behaviour in distribution networks [24]. While such effort can be accurate at medium voltage (MV) level due to the aggregation of load points, the LV load points are directly dependent on irrational consumer behaviour, making load modelling challenging and impeding the implementation of DSSE in existing ICT infrastructure. Furthermore, state of the art in distribution network monitoring, requires representation of active and reactive power at each load point in the network [20]. With the General Data Protection Regulation (GDPR) activated by the European Union (EU), consumers have more control over their personal data, including the consumption data from residential smart meters [25]. A complete set of smart meter data is therefore not guaranteed for the execution of the DistFlow or DSSE methods.

Considering the limitations in current state of the art in DistFlow and DSSE, the work presented in this paper propose a novel method that can estimate network voltage conditions without complete datasets and without utilizing consumer consumption data. The minimum amount of data required by the proposed algorithm is three-phased voltage magnitude measurements from one arbitrary node in the feeder, however, voltage readings from additional nodes can be included to narrow the estimated voltage interval of the entire feeder. Since the algorithm can execute with one measurement, issues with ICT infrastructure performance are overcome. Furthermore, the proposed method only considers the per phase voltage magnitude, which does not directly reveal the consumption pattern of the consumer.

The proposed method is based on knowledge of the load connection and connected equipment, and provides an estimation of the voltage interval of a radial feeder. As distribution networks have high $\mathrm{R} / \mathrm{X}$ ratios, the complex current has an impact on the worst case voltage magnitudes in the network. Therefore, the proposed method includes an estimation of worst case line current magnitude and angle in each line segment of the LV feeder, where the worst case conditions are defined as those that cause the largest deviation in voltage magnitude across the conductor. Utilizing the worst case current interval, the methodology estimates an interval of all possible voltage magnitudes at all nodes and phases of the feeder. The method differentiates from existing distribution network monitoring methods, as it is capable of estimating the network conditions using the existing ICT infrastructure, does not require time synchronism of measurements, and considers data privacy concerns regarding consumption information from smart meters.

The rest of the paper is organized as follows. Section 2 describes the proposed voltage interval estimation method, including the estimation of worst case current magnitude and angle boundaries. In Section 3 a test feeder is described together with an analysis of the results from case studies. Section 4 discuss the possible application of the proposed method, and Section 5 concludes.

\section{Methodology}

The proposed methodology for voltage magnitude interval estimation is intended for application in radial distribution networks. Here, especially at a LV level, unbalanced operation is a possible and likely scenario. Therefore, the proposed method considers all three phases.
An illustration of the method initialization and execution is shown in Fig. 1.

Execution of the estimation method is initiated with the acquisition of a voltage magnitude measurements from an arbitrary node within the feeder as shown in Fig. 1. With the existing deployment of smart meters, forecasted to reach 1 billion devices globally in 2022 [26], and their configuration capabilities, these devices are considered suitable as data sources for the proposed method. Besides the acquisition of voltage measurements, the proposed method is based on feeder information which is included in the initialization phase. The feeder information must therefore be represented with a specific syntax as described in Section 2.1.

In addition to the specific feeder information, the proposed method requires an estimation of the worst case current interval of each conductor as illustrated in Fig. 1. These are estimated through the methodology described in Section 2.2. In this work, the worst case current is that which causes the most severe change in voltage magnitude across the conductor. Therefore, the worst case current magnitude is described by its maximum in either direction through a conductor, and determined by the distribution of connected loads in the feeder as well as the topology of the feeder as described in Section 2.2.1.

In addition, the angle of the conductor current has a considerable impact on the voltage. This is illustrated in Fig. 1 through the included complex current from the worst case current estimation. The definition of the worst case current angle is equal to that of the magnitude. Its estimation process is described in Section 2.2.2.

With an overview of the worst case current interval in polar form for each feeder conductor, the interval of feeder voltage magnitudes can be estimated as measurements are received. The proposed calculation method, shown in the dark grey box in Fig. 1, executes based on the received reading and rated accuracy of the meters. The resulting interval encapsulates the actual voltage profile, and the entire estimation process is described in details in Section 2.3 .

\subsection{Feeder information}

For convenience, the feeder information is represented using matrix notation. The first matrix is a bus incidence matrix and contains information about the connection between lines and nodes in the network. Each row in the $L N$-matrix represent a line in the feeder, and each column in the matrix represent a node. $L N$ is therefore a $N_{l} \times N_{n}(0,1)$-matrix, where $N_{l}$ is the number of lines in the feeder and $N_{n}$ is the number of nodes. An element $(r, c)$, in the LN matrix, is equal to 1 when representing a connection between the $r$ 'th line and $c^{\prime}$ th node.

The second matrix of a particular feeder is defined as the $L L$-matrix. This matrix is a $N_{l} \times N_{n}(0,1)$-matrix, where each row represents each line and each column represents each node. This matrix describes the

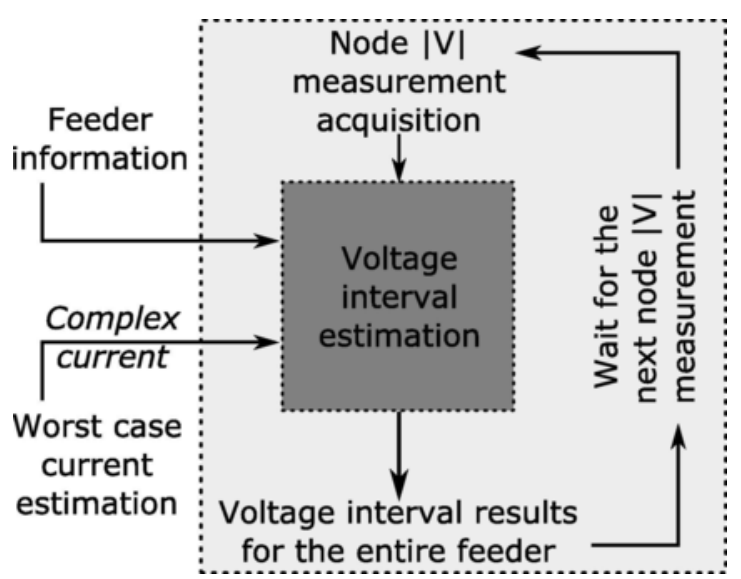

Fig. 1. Flowchart describing the execution of the proposed methodology. Information outside the light grey box only needs update in case of network configuration changes. 
shortest path of lines connecting a node to the root-node. In this way, if the $c^{\prime}$ th node connects to the root-node through lines 1,2 and 3, the three first rows in column $c$ are equal to 1 while all other elements in the column are equal to 0 .

\subsection{Line current interval estimation}

With the interval arithmetic approach described in [12], the line current intervals are expressed through a minimum, $P_{l p}^{\min }$, and a maximum $I_{l p}^{\max }$ for each line segment $l \in N_{l}$ and each of the three phases $p$. The outcome of the first step of the proposed method is likewise a current interval matrix $I_{a b c}^{w c}$ for each line segment $l$ and phase $p$ as shown in (1).

$I_{a b c}^{w c}=\left[\begin{array}{lllllll}I_{1 a}^{\min } & I_{1 b}^{\min } & I_{1 c}^{\min } & \ldots & I_{N_{l} a}^{\min } & I_{N_{l} b}^{\min } & I_{N_{l} c}^{\min } \\ I_{1 a}^{\max } & I_{1 b}^{\max } & I_{1 c}^{\max } & \ldots & I_{N_{l} a}^{\max } & I_{N_{l} b}^{\max } & I_{N_{l^{c}}}^{\max }\end{array}\right]^{T}$

\subsubsection{Line current magnitude interval}

Estimating the magnitude of the line current intervals in (1) utilize prior knowledge of network and load point information. Considering the network in Fig. 2 with an external grid connection at node $N 1$, and consuming units connected to nodes $N 2$ to $N 7$, and conductors connecting the nodes.

The maximum line current to be expected in the line segment between nodes $N 1$ and $N 2$ is estimated as the sum of maximum phase currents in each of the consumers connected to node downwards from node $N 2$. That is, the combined maximum current from loads at nodes $N 2$ to $N 7$. This estimation can be done for all the conductors in the radial feeder using the $L L$-matrix formed in Section 2.1, and the feeder load point limits.

The load point information is assumed known through an overview of large consuming equipment, e.g. EVs and heat pumps (HPs), and general residential consumption limited by fuses, i.e. $13 \mathrm{~A}$ per phase in Denmark. Therefore, a matrix containing the load limit of current draw per phase and node can be constructed as in (2).

$\left|I_{n a b c}^{\max }\right|=\left[\begin{array}{ccc}\left|m_{1 a}^{\max }\right| & \cdots & \left|I_{N_{n} a}^{\max }\right| \\ \left|m_{1 b}^{\max }\right| & \cdots & \left|I_{N_{n} b}^{\max }\right| \\ \left|I_{1 c}^{\max }\right| & \cdots & \left|I_{N_{n} c}^{\max }\right|\end{array}\right]$

The expected maximum current in each line $l$ and phase $p$, represented by $\left|I_{l p}^{\max }\right|$ in (1) can be calculated by transforming the results of (3) into a column vector of length $3 \cdot N_{l}$.

$\left|I_{l a b c}^{\max }\right|=\left|I_{n a b c}^{\max }\right| \cdot L L^{T}$

\section{External grid}

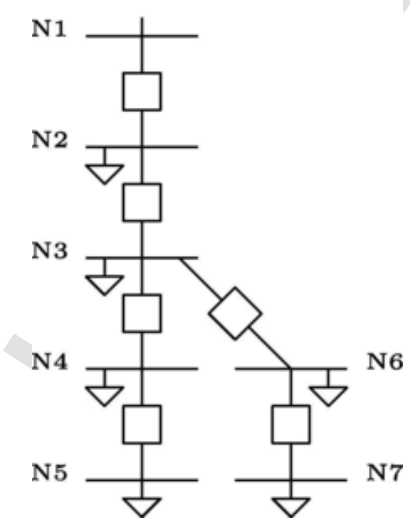

Fig. 2. Simple two node system.
The minimum line current magnitude is found in a similar way as for the maximum, and $\left|I_{l p}^{\min }\right|=0$ if all connected load points are purely consumption. However, modern distribution networks can be bidirectional, meaning $\left|I_{l p}^{\mathrm{min}}\right|>0$, with a negative current direction, if a generating unit is connected to phase $p$. As for the estimation of maximum line current, it is assumed that the network operator knows about connected units capable of injecting current into the LV network, e.g. PV plants and batteries.

\subsubsection{Line current angle interval}

Estimating the current angle intervals for each phase and each line segment is more complicated than for the magnitude as it depends on the specific line impedance matrix $Z_{l}$. This is further complicated by the inclusion of a neutral conductor and the geometry of LV network cables and overhead lines, making the impedance matrix non-bisymmetrical. The three-phase conductors will therefore experience different voltage drop at equal current angles. Furthermore, the worst case current angle combination depends on the combination of current magnitudes for the specific line segment.

Therefore, estimation of the current angle interval, resulting in the most severe voltage drop across a line segment, can be done through solving a non-linear optimization problem considering the current magnitudes intervals estimated in Section 2.2.1. Solving this optimization problem, gives the most severe and therefore optimal combination of current angles of a line segment.

To simplify the estimation of the current angle interval, the two optimization problems are combined as a single minimization problem. This is possible since the current direction simply changes the sign of the voltage change at the receiving end. Therefore, an overall objective function $f$ can be formulated for each line $l$ and extreme $e$ (minimum or maximum), using Ohm's law as shown in (4), subject to the constraints described in (5), which ensures a single solution to the non-linear programming problem.

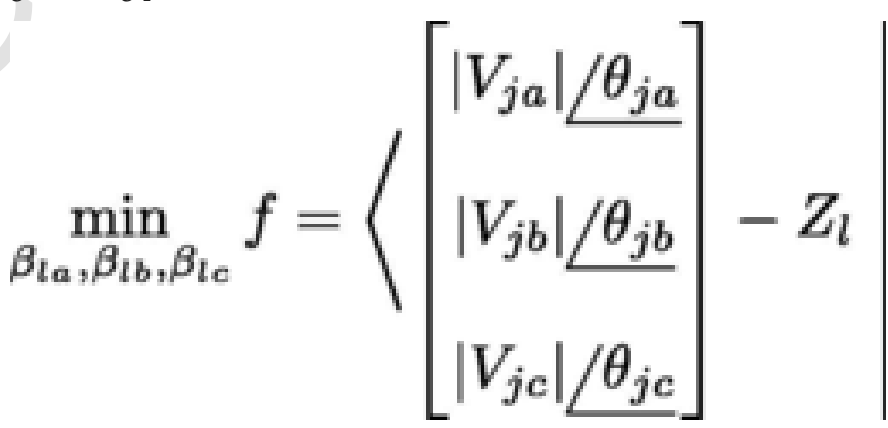

Subject to

$-\frac{\pi}{2} \leqslant \beta_{l p} \leqslant \frac{\pi}{2} \quad \forall_{p \in\{a, b, c\}, l \in N_{l}}$

where $\left|I_{l p}^{e}\right|$ and $\beta_{l p}^{e}$ are the line $l$, extreme $e$, phase $p$ current magnitude and angle, respectively, and $\left|V_{j p}\right|$ and $\theta_{j p}$ are the node $j$, phase $p$ voltage magnitude and angle, respectively. The sending end com-

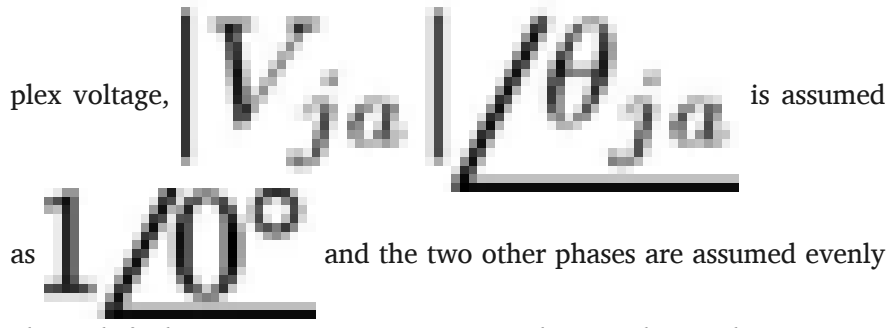

phase shifted $120^{\circ}$. For convenience in evaluating the resulting current angles, the voltage phase angels are added in the phase current angles in (4). A magnitude imbalance at the sending end has no impact on the 
optimal solution, only the objective value in (4), and the three phases are therefore assumed to have balanced magnitudes.

An imbalance in the sending end voltage angle however will affect the optimal solution. This effect is however only limited while considering a maximum allowed voltage unbalance factor (VUF) of $2 \%$ according to EN 50160 [27]. The sending end voltage angle is assumed balanced in the proposed method, and the impact of this assumption is evaluated in Section 3 through a probabilistic sensitivity analysis.

The optimization problem in (4) and (5) is solved twice for each line segment, once for each interval extreme. This gives the needed quantities in the phasor representation of the line current interval matrix in (1). Since the interval matrix only change due to changes in the load composition and long term degradation of feeder infrastructure from partial discharges and other phenomenons, this part of the methodology only requires long-term updating.

\subsection{Voltage magnitude assessment}

The voltage magnitude interval describes the estimated extremes $\left|V_{n p}^{\min }\right|$ and $\left|V_{n p}^{\max }\right|$ for each node $n$ and phase $p$. These results are the output of the proposed method and are structured as in (6).

$\left|V_{a b c}^{w c}\right|=\left[\begin{array}{lllllll}\left|V_{1 a}^{\min }\right| & \left|V_{1 b}^{\min }\right| & \left|V_{1 c}^{\min }\right| & \cdots & \left|V_{N_{n} a}^{\min }\right| & \left|V_{N_{n} b}^{\min }\right| & \left|V_{N_{n} c}^{\min }\right| \\ \left|V_{1 a}^{\max }\right| & \left|V_{1 b}^{\max }\right| & \left|V_{1 c}^{\max }\right| & \cdots & \left|V_{N_{n} a}^{\max }\right| & \left|V_{N_{n} b}^{\max }\right| & \left|V_{N_{n} c}^{\max }\right|\end{array}\right]^{T}$

The two matrices $L N$, and $L L$ are central in the proposed algorithm and only depend on the feeder information. When considering acquiring an arbitrary node measurement, additionally one vector and one matrix are formed to estimate the feeder voltage intervals.

The vector $N$ is constructed and used to index the location of the received measurement. Therefore, this vector is of size $N_{n} \times 1$ and contains $N_{n}-1$ zeros and a single 1 element. The non-zero element represents the node under consideration, which changes as the algorithm advances.

With the vector $N$, a diagonal matrix $C L$ can be formed, which defines the lines connected to that node. The matrix $C L$ is calculated using (7).

$C L=\operatorname{diag}(L N \cdot N)$

\subsubsection{Algorithm flow}

The proposed algorithm is developed for execution each time an arbitrary node voltage magnitude reading is acquired by the control center from node $n_{a c q}$. For the acquired measurement, the sensor accuracy classification is given by datasheets according to standards such as IEC 62053. This error classification is used to form an interval by adding and subtracting the indicated measurement error to the acquired voltage magnitude reading. This gives the initial interval at the node $n_{a c q}$ with the boundaries $\left|V_{n_{a c q}}^{\min }\right|$ and $\left|V_{n_{a c q} p}^{\max }\right|$. The acquisition initiates the algorithm described in Algorithm 1, by increasing the size of the indexation vector $N_{i d x}$ to one, with the element being equal to the $n_{a c q}$ node number. The role of the indexation vector is to control the sequence of node voltage estimations from the measurement node $n_{a c q}$ and ensure that all nodes are estimated.

Algorithm 1. Estimate complete voltage magnitude interval matrix from single node reading

1: while $N_{i d x}$ contains at least 1 element do

Initialize the $N$-vector of zeros

$N\left(N_{i d x}(1)\right) \leftarrow 1$

Calculate $C L$ using (7)

for all Rows $c l \in C L d o$

if $\|c l\|_{2}>0$ then

$l_{u c} \leftarrow c l \cdot L N$

$l_{u c}\left(N_{i d x}(1)\right) \leftarrow 0$

$n_{n b n} \leftarrow$ index of non-zero element $\in l_{u c}$

if $n_{n b n}$ has not yet been estimated then

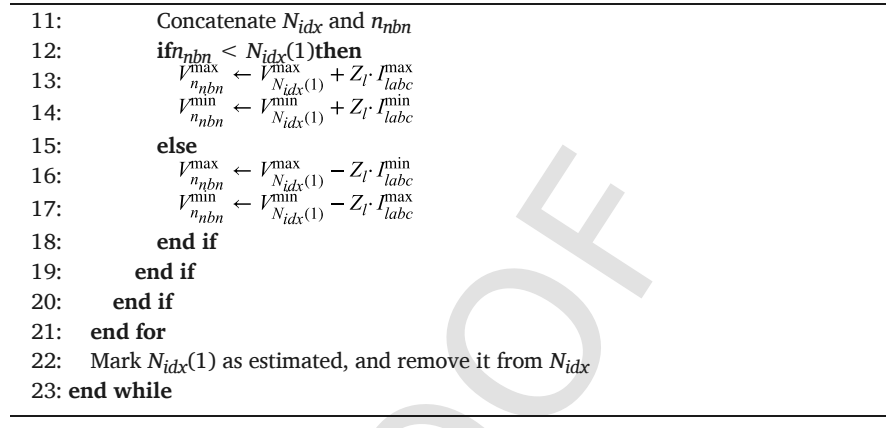

The idea behind the algorithm is to start from the node of the acquired measurement and identify the lines connected to this node. This process is described in lines 2 to 4 in Algorithm 1.

For each line under consideration $l_{u c}$, the two nodes connected to the line are identified in line 7 of Algorithm 1 . Then the node corresponding to $N_{i d x}(1)$ is removed from the line under consideration, enabling an identification of the neighbouring node $n_{n b n}$, described in lines 8 and 9 of Algorithm 1.

The if-statement in line 12 is used to determine whether the $n_{n b n}$ -node is closer to the root-node than the $N_{i d x}(1)$-node, through examining the indexation. This entails that the indexation of nodes in the network must increase away from the root node.

\section{Case studies}

The proposed method is tested using a modified version of an European LV feeder benchmark system. The European LV benchmark feeder network from Cigré is considered and shown in Fig. 3[28]. An apparent power base per phase of $S_{\text {base }}=166.67 \mathrm{kVA}$ and a line to neutral voltage base of $V_{\text {baseLN }}=230.94 \mathrm{~V}$ is used.

All load points in the feeder of Fig. 3 are residential. In modern European households, typical fuse ratings are at $13 \mathrm{~A}$ per phase, meaning each household will have an absolute max current draw of 39 A equal to approximately $9 \mathrm{~kW}$. The apparent power and power factor rating of the original and modified LV feeders are given in Table 1 . The modifications of the Cigré feeder are based on the distribution of the node $\mathrm{R} 1$ consumption to the remaining load points. This modification distributes the branch current loading within the network and thereby entails

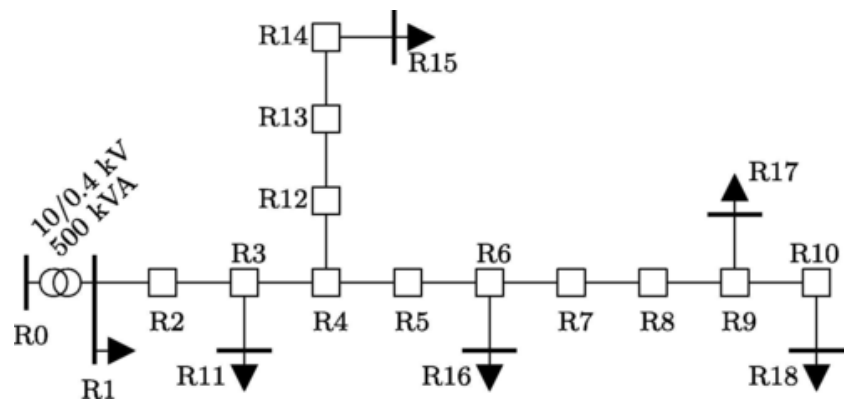

Fig. 3. Cigré European LV benchmark network.

Table 1

Original and modified ratings of load points in Cigré network.

\begin{tabular}{lllllllll}
\hline Node & & 1 & 11 & 15 & 16 & 17 & 18 & All \\
\hline$|S|[\mathrm{kVA}]$ & Original & 200 & 15 & 52 & 55 & 35 & 47 & 404 \\
& Modified & 0 & 54 & 90 & 90 & 72 & 81 & 387 \\
$\cos (\phi)$ & & 0.95 & 0.95 & 0.95 & 0.95 & 0.95 & 0.95 & \\
Houses & Original & 22.2 & 1.7 & 5.8 & 6.1 & 3.9 & 5.2 & 44.9 \\
& Modified & 0 & 6 & 10 & 10 & 8 & 9 & 43 \\
\hline
\end{tabular}


a distribution of the feeder voltage drops. For each of the remaining load points, the original number of houses are estimated using the prior assumed maximum residential consumption, and four additional houses at each load point are implemented. This means the total consumption in the modified network correspond to $96 \%$ of the original network.

Besides differences in the load point configurations, the LV grid in Fig. 3 contains two different conductors. The lines connecting nodes R1 to $\mathrm{R} 10$ are all referred to as type 1 , while the remaining lines are of type 2 . The main difference between these conductors is apparent from [28], where the cross sectional area of the two conductors is 240 and $50 \mathrm{~mm}^{2}$, respectively.

The LV feeder is used to execute three case studies, each with specific objectives.

1. Sensitivity analysis of parameters affecting the worst case line currents from a voltage deviation perspective.

2. Illustrate the interval estimation from single node measurements and evaluate the granularity expected from the proposed methodology while using multiple measurements within a short time period.

3. Evaluate the proposed method application considering different meter distribution and accuracy scenarios.

\subsection{Sensitivity analysis}

For the first case study, the proposed worst case complex current is identified for each line in the Cigré feeder using the optimization described in Section 2.2.1 and the genetic algorithm (GA) solver. The resulting phasor diagram is given in Fig. 4(a).

From Fig. 4(a) the dominating parameter in determining the worst case current angle is the line impedance matrix while the current magnitude has only a limited effect. This can further be used to simplify the method application by only estimating the current angles for lines with equal impedance regardless of the current magnitude.

In the proposed method, the voltage angles at the sending end are assumed balanced. Here we can analyse this assumption through Monte Carlo simulations where the sending end voltage angle is randomized and unbalanced. The outcome of the probabilistic analysis is the correlation between imbalance and the average receiving end voltage drop experienced using a constant line current and balanced sending end voltage magnitude. The Monte Carlo simulations are conducted for a representation of each of the conductor types in the network as shown in Fig. 4(b).

The results in Fig. 4(b) further include the voltage drop during a simplified situation where zero current phase shift is assumed. The difference in conductor geometry results in higher $\mathrm{R} / \mathrm{X}$ ratios of type 2

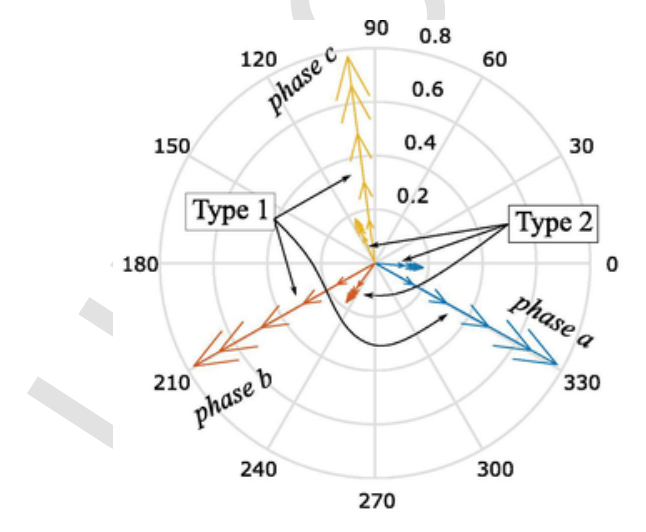

(a) Phasor diagram of worst case line

currents. than type 1 , this consequently affects the voltage drop sensitivity to the current angle where a lower $\mathrm{R} / \mathrm{X}$ ratio gives larger difference between the proposed method and the simplification. Furthermore, the distribution from the probabilistic analysis indicate how a smaller R/X-ratio increases the voltage magnitude deviation sensitivity to the current angle. Finally, Fig. 4(b) show that within the allowed VUF in distribution networks according to EN 50160, the voltage magnitude deviation has limited sensitivity towards the unbalance of the voltage angles at the sending node in Fig. 2.

\subsection{Assessment granularity}

The benefits of the proposed method can be evaluated by estimating the voltage intervals based on a measurement acquisition from 1 smart meter at each of the load points, assuming each smart meter measures and reports the voltage magnitude for each of the three phases of the LV feeder. The evaluation is demonstrated using randomized operating conditions with the apparent power and inductive power factor of each load point and phase in Table 2.

With the load properties in Table 2, a power flow calculation is executed to retrieve the voltage magnitude at each node and phase using the DistFlow method described in [10], which is used as a representation of the actual voltage profile of the feeder during the loading described in Table 2. For each load point in Fig. 3, the voltage magnitude measurements of all phases are distorted by adding a random normal distributed error with zero mean and standard deviation, $\sigma=0.01 / 3$ . The standard deviation of the measurement error is based on an assumed meter accuracy of $\pm 1 \%$ and that $3 \cdot \sigma$ encapsulates $99.7 \%$ of the normal distributed probability density function. The distorted values are used in the execution of Algorithm 1 and the resulting voltage assessment for phase a is presented in Fig. 5.

Each of the load points in the modified feeder of Fig. 3 are highlighted in Fig. 5 by a gray ellipse with a colored edge. These ellipses illustrates and encapsulates the voltage magnitude measurement interval based on the acquired reading at the respective nodes. For each measurement point, a maximum and minimum voltage profile is estimated and shown in Fig. 5. This way, Fig. 5 shows how a single measurement point gives a rough estimate of the voltage profile across entire feeder, where it is narrowest at the point of measurement shown by the grey ellipses. The assessment based on a single measurement offers value by narrowing the possible operational region of voltage magnitudes. Observe how a measurement at node R11 limits the operational boundary within the EN 50160 limits of $\pm 10 \%$ error from rated voltage.

In addition, Fig. 5 illustrates the value of utilizing the proposed method when acquiring multiple measurements with small time delays. This is shown by the estimation of voltage magnitude interval profiles

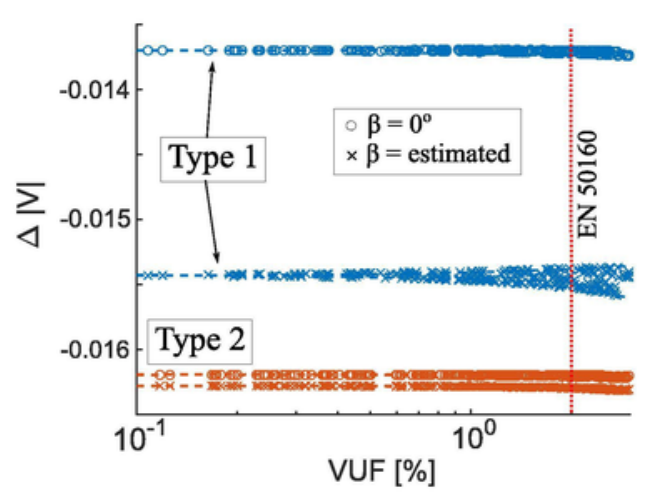

(b) VUF impact on angle estimation and consequently voltage drop.

Fig. 4. Sensitivity analysis of parameters affecting the worst case line current estimation. 
Table 2

Load point phase parameter during voltage assessment evaluation.

\begin{tabular}{|c|c|c|c|c|c|c|}
\hline Node & & 11 & 15 & 16 & 17 & 18 \\
\hline \multirow{3}{*}{$|S|[\mathrm{kVA}]$} & Phase a & 17.4 & 29.0 & 50.5 & 21.0 & 70.8 \\
\hline & Phase b & 16.8 & 28.0 & 85.8 & 43.8 & 64.0 \\
\hline & Phase b & 12.2 & 20.3 & 64.3 & 52.6 & 6.1 \\
\hline \multirow{3}{*}{$\cos (\phi)$} & Phase a & 0.94 & 0.99 & 1.00 & 0.95 & 0.96 \\
\hline & Phase b & 0.92 & 0.97 & 0.92 & 0.94 & 0.90 \\
\hline & Phase b & 0.95 & 0.98 & 0.99 & 0.96 & 0.96 \\
\hline
\end{tabular}

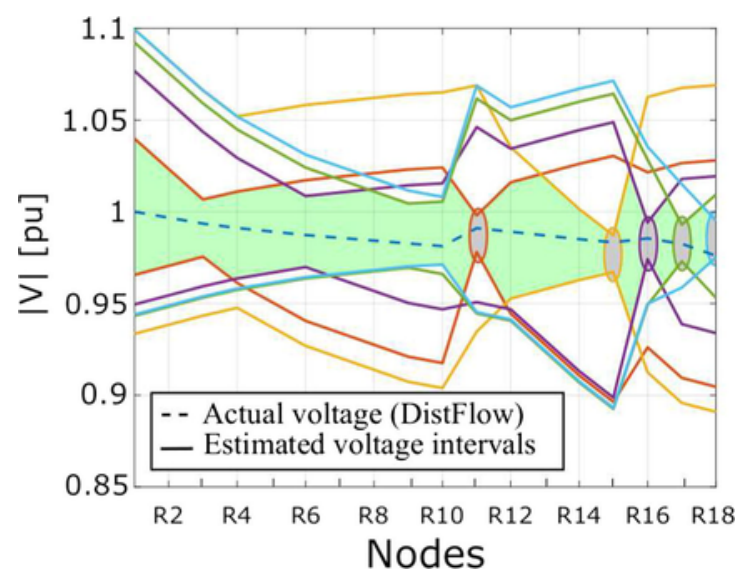

Fig. 5. Phase a voltage magnitude assessment with load configuration in Table 2. Each set of colored voltage magnitude profiles illustrate an estimation from acquiring a measurement from each of the load points, R11, R15, R16, R17, and R18 are shown using red, yellow, purple, green, and light blue lines, respectively. (For interpretation of the references to colour in this figure legend, the reader is referred to the web version of this article.)

for each of the five load points, (R11, R15, R16, R17, and R18). Highlighting the region within the interval boundaries of all estimated profiles in green in Fig. 5, shows how it encapsulates the DistFlow resulting voltage magnitude profile. Such voltage interval estimation enables the operator to easily and quickly assess the need for more in-depth evaluation of the feeder seen from a voltage security perspective.

The results in Fig. 5 also show the strength of the proposed method in conditions where the ICT infrastructure is unable to transfer measurements in a realible fashion. Such ability is visible by considering each of the colored interval estimations in Fig. 5. If only a single measurement is received, an interval of the feeder voltage profile will still be estimated and transmitted to the operator. This way, the proposed method can utilize minimal measurement acquisition and still inform the network operator on the operational conditions within the feeder.

\subsection{Application and performance}

Evaluating the performance of the proposed method is done through a probabilistic analysis of the method's ability to capture the voltage profile from DistFlow results within the estimated interval. To perform this analysis, Monte Carlo simulations with a population of 100,000 are performed, where the power flow is calculated using the
DistFlow method described in [10], and the voltage intervals are estimated using the proposed method. For this analysis the voltage interval estimation is performed through assuming an acquisition of measurements from multiple meters within a short time period. The number of measurement locations, and thereby number of estimated voltage intervals for each of the Monte Carlo simulations is based on scenario specific meter distributions. Furthermore, the assumed meter accuracy is varied for evaluating the the method's dependency on meter quality.

For the distribution of meters, and thereby the number of estimated intervals, three scenarios are considered, one where meters are placed at the nodes furthest away from the root node, that is at R15 and R18, one where meters are placed at all load points R11, R15, R16, R17, and R18 in Fig. 3, and one where meters placed at all nodes R1 to R18 in the feeder. For the second and third meter acquisition scenarios, DSSE could theoretically be implemented, however this requires handling power consumption or node voltage angle information which is not considered available for the proposed methodology. Furthermore, three different meter accuracy conditions are assumed, where all meters have an accuracy of $\pm 1 \%, \pm 0.1 \%$, and $\pm 0.01 \%$, respectively.

To execute the probabilistic analysis, the apparent power of the feeder load points is randomized within the rated apparent power given in Table 1, and through an assumption that all residential load points have integrated distributed generation through rooftop PV equivalent to $30 \%$ of their rated consumption. Additionally, the power factor is randomized between 0.9 lagging and 0.9 leading. The results are analysed by looking at instances where the voltage profile from the DistFlow results, is outside the narrowest estimated voltage interval considering an estimation using measurements from multiple meters according to the meter distribution scenario. The violation of the assessed boundaries are registered per phase and node, meaning the total count of evaluations is $3 \cdot 18 \cdot 100,000=5,400,000$. The results of the probabilistic analysis are given in Table 3.

The results in Table 3 show that there is a small risk of violating the assessed voltage boundaries, meaning the proposed method is able to fully encapsulate the voltage profile of the feeder in most cases. This risk of wrongful estimation is evaluated to depend on the number of measurement points collected, but is below $1 \%$ for all the different distribution scenarios. The inability to encapsulate the voltage profile is partly due to the conversion of the measurement error the sensor classification to an interval, which not encapsulates $100 \%$ of the possible error. Furthermore, the assumed balanced sending end voltage when estimating the current angle entails a minor error as described in Section 3.1. Finally, the nonlinearity of the optimization problem, the objective of finding the worst current angle based on an average voltage deviation for all three phases, and the imperfect accuracy of the genetic algorithm all contributes to the violations.

Looking at the violation statistics of Table 3, it is seen that having highly accurate sensors and a high performance of the data communication infrastructure supports the proposed method by limiting the median and maximum violations. The highest violation is seen when measurements are acquired from all load points and all sensors have high rated measurement error. Here however, the violation is a most $1.8 \mathrm{~V}$ which is less than 0.01 per unit. This extreme case is not common as can be seen by the median which is around a tenth of the maximum observed violation.

Table 3

Probabilistic analysis of voltage estimation violations in different meter distribution and accuracy scenarios.

\begin{tabular}{|c|c|c|c|c|c|c|c|c|c|}
\hline \multirow{2}{*}{$\begin{array}{l}\text { Meters at } \\
\text { Meter accuracy }\end{array}$} & \multicolumn{3}{|c|}{$\mathrm{R} 15$ \& R18 } & \multicolumn{3}{|c|}{$\mathrm{R} 11, \mathrm{R} 15, \mathrm{R} 16, \mathrm{R} 17$ \& R18 } & \multicolumn{3}{|c|}{ R1-R18 } \\
\hline & $\pm 1 \%$ & $\pm 0.1 \%$ & $\pm 0.01 \%$ & $\pm 1 \%$ & $\pm 0.1 \%$ & $\pm 0.01 \%$ & $\pm 1 \%$ & $\pm 0.1 \%$ & $\pm 0.01 \%$ \\
\hline \multirow[t]{2}{*}{ Count } & 1617 & 1650 & 1637 & 4044 & 4041 & 3984 & 14560 & 14719 & 15485 \\
\hline & $0.03 \%$ & $0.03 \%$ & $0.03 \%$ & $0.07 \%$ & $0.07 \%$ & $0.07 \%$ & $0.27 \%$ & $0.27 \%$ & $0.29 \%$ \\
\hline Median $[\mathrm{mV}]$ & 152 & 15.3 & 1.56 & 155 & 15.6 & 1.65 & 156 & 15.8 & 1.68 \\
\hline $\operatorname{Max}[\mathrm{mV}]$ & 1446 & 127 & 38.1 & 1553 & 155 & 41.8 & 1788 & 177 & 325 \\
\hline
\end{tabular}




\section{Application in modern LV networks}

The proposed method considers each of the three phases and the coupling between them, and can be implemented for feeders where line segments are less than three-phased. With an estimation of the voltage magnitudes in each of the phases of a radial distribution feeder, the network operator can operate and plan the load and network expansion more accurately, e.g. improving phase distribution of new residential equipment. The potential unbalance between the phases can also be supervised and controlled in long term.

The proposed method can also assist the network operator by indicating potential risks where investments are necessary to improve overall security of the feeder. Since the proposed method requires few measurements, it can be considered to run continuously and thereby further help operating the system by determining different control actions, e.g. reconfiguration or voltage compensation.

Observing the critical LV feeders requires the acquisition of measurements. With the proposed method, smart meters are considered an ideal acquisition device, since it is distributed within the network and is customizable in its communication of measurements. For continuous visibility of the network operation, the proposed method could be used to incorporate asynchronized meter readings, or synthesize them in a period, to gain higher accuracy. Furthermore, if the proposed method identifies a feeder with very high voltage magnitude range for the majority of measurement nodes, possible investments in the ICT infrastructure could be identified and suggested.

\section{Conclusion}

Considering ICT infrastructure reliability limitations and issues with data privacy of smart meter readings, this work aims at estimating the voltage magnitudes in LV distribution feeders using existing ICT infrastructure. A methodology based on knowledge of load point equipment, and thereby line current intervals, is proposed. The methodology use the idea of interval arithmetic, and with the acquisition of voltage magnitudes from a single node in the LV feeder, the proposed method can estimated the phase voltage magnitude interval boundaries for all nodes in the feeder.

The line current interval estimation is evaluated through a sensitivity analysis, where the impedance matrix of the conductor is found to have a greater impact on the worst case current estimation, than the current magnitude through the conductor. Furthermore, the assumption of balanced sending-end voltage used in the optimization is evaluated and results show that only a minor impact is expected while considering the EN 50160 standard requirements to the voltage unbalance factor.

A randomized load configuration and the corresponding power flow results show the value of the proposed method. From this analysis, the proposed method is shown to give the operator a means of evaluating the voltage magnitude profile of a feeder through acquisition from a single measurement point. Furthermore, the results indicate the benefits of performing the proposed voltage assessment on multiple measurements acquired with small delays. This utilization narrows the voltage magnitude boundaries giving the operator a more detailed view of the operation during quasi steady state operation.

The proposed method is evaluated using Monte Carlo simulations and an analysis of the violations of the estimated boundaries. Although the proposed method is unable to perform perfectly in any of the three considered meter distributions and rated error scenarios, the method encapsulates the voltage magnitude profile in more than $99 \%$ of the simulated conditions. With these findings, possible areas of application are discussed, including optimal asynchronism of meter acquisition, per phase implementation planning, feeder risk assessment and identification of critical feeders, and ICT investment planning.

\section{Appendix A. Supplementary material}

Supplementary data associated with this article can be found, in the online version, at https://doi.org/10.1016/j.ijepes.2019.05.041.

\section{References}

[1] IEC Standard for Communication Networks and Systems in Substations. IEC 61850. 1st ed.; 2003-04

[2] M. Kuzlu, M. Pipattanasomporn, S. Rahman, Communication network requirements for major smart grid applications in HAN, NAN, and WAN, J Com Net 67 (2014) 74-88, https://doi.org/10.1016/j.comnet.2014.03.029.

[3] V.C. Gungor, D. Sahin, T. Kocak, S. Ergut, C. Buccella, C. Cecati, et al., A survey on smart grid potential applications and communication requirements, IEEE Trans Ind Inf 9 (2013) 28-42, https://doi.org/10.1109/TII.2012.2218253.

[4] X. Yu, Y. Xue, Smart grids: A cyber-physical system perspective, Proc IEEE 104 (2016) 1058-1070, https://doi.org/10.1109/JPROC.2015.2503119.

[5] P.G. Da Silva, D. Illić, S. Karnouskos, The impact of smart grid prosumer grouping on forecasting accuracy and its benefits for local electricity market trading, IEEE Trans Smart Grid 5 (2014) 402-410, https://doi.org/10.1109/TSG.2013.2278868.

[6] R. Singh, B.C. Pal, R.B. Vinter, Measurement placement in distribution system state estimation, IEEE Trans on Power Syst 24 (2009) 668-675, https://doi.org/10. 1109/TPWRS.2009.2016457.

[7] J. Liu, J. Tang, F. Ponci, A. Monti, C. Muscas, P.A. Peforaro, Trade-offs in PMU deployment for state estimation in active distribution grids, IEEE Trans Smart Grid 3 (2012) 915-924, https://doi.org/10.1109/TSG.2012.2191578.

[8] M. Baran, F.F. Wu, Optimal capacitor placement on radial distribution systems, IEEE Trans Power Deliv 4 (1989) 725-734, https://doi.org/10.1109/61.19265.

[9] M. Baran, F.F. Wu, Optimal sizing of capacitors placed on a radial distribution system, IEEE Trans Power Deliv 4 (1989) 735-743, https://doi.org/10.1109/61. 19266

[10] C.S. Cheng, D. Shirmohammadi, A three-phase power flow method for real-time distribution system analysis, IEEE Trans Power Syst 10 (1995) 671-679, https:// doi.org/10.1109/59.387902.

[11] M. Aien, A. Hajebrahimi, M. Fotuhi-Firuzabad, A comprehensive review on uncertainty modeling techniques in power system studies, Renew Sustain Energy Rev 57 (2016) 1077-1089, https://doi.org/10.1016/J.RSER.2015.12.070.

[12] B. Das, Consideration of input parameter uncertainty in load flow solution of three-phase unbalanced radial distribution system, IEEE Trans Power Syst 21 (2006) 1088-1095, https://doi.org/10.1109/TPWRS.2006.876698.

[13] A. Primadianto, C.N. Lu, A review on distribution system state estimation, IEEE Trans Power Syst 2 (2017) 3875-3883, https://doi.org/10.1109/TPWRS.2016. 2632156.

[14] Hayes B, Prodanovic M. State estimation techniques for electric power distribution systems. In: European modelling symposium 2014. Pisa, Italy; 2014.

[15] I. Roytelman, S.M. Shahidehpour, State estimation for electric power distribution systems in quasi real-time conditions, IEEE Trans Power Deliv 8 (1993) 2009-2015, https://doi.org/10.1109/61.248315.

[16] M.E. Baran, A.W. Kelley, A branch-current-based state estimation method for distribution systems, IEEE Trans Power Syst 10 (1995) 483-491, https://doi.org/10. $1109 / 59.373974$

[17] A.P. Meliopoulos, F. Zhang, Multiphase power flow and state estimation for power distribution systems, IEEE Trans Power Syst 11 (1996) 939-946, https://doi.org/ $10.1109 / 59.496178$.

[18] A. Alimardani, F. Therrien, D. Atanackovic, J. Jatskevich, E. Vaahedi, Distribution system state estimation based on nonsychronized smart meters, IEEE Trans Smart Grid 6 (2015) 2919-2928, https://doi.org/10.1109/TSG.2015.2429640.

[19] M.M. Albu, M. Sănduleac, C. Stănescu, Syncretic use of smart meters for power quality monitoring in emerging networks, IEEE Trans Smart Grid 8 (2017) 485-492, https://doi.org/10.1109/TSG.2016.2598547.

[20] S. Bhela, V. Kekatos, S. Veeramachaneni, Enhancing observability in distribution grids using smart meter data, IEEE Trans Smart Grid (2017) https://doi.org/10. 1109/TSG.2017.2699939, EA.

[21] Luan W, Sharp D, LaRoy S. Data traffic analysis of utility smart metering network In: IEEE PES GM 2013. Vancouver, BC, Canada; 2013.

[22] S. Repo, F. Ponci, D.D. Giustina, A. Alvarez, C.C. Garica, Z. Al-Jassim, et al., The IDE4L project: Defining, designing, and demonstrating the ideal grid for all, IEEE Power Energ Mag 15 (2017) 41-51, https://doi.org/10.1109/MPE.2017.2662329.

[23] Commission for Energy Regulation, Electricity smart metering technology trials findings report. Information paper, May 2011

[24] K. Dehghanpour, Z. Wang, J. Wang, Y. Yuan, F. Bu, A survey on state estimation techniques and challenges in smart distribution systems, IEEE Trans Smart Grid 10 (2019) 2312-2322, https://doi.org/10.1109/TSG.2018.2870600.

[25] A. Albert, R. Rajagopal, Smart meter driven segmentation: What your consumption says about you, IEEE Trans Power Syst 28 (2013) 4019-4030, https://doi.org/10. 1109/TPWRS.2013.2266122.

[26] Strother N, Lockhart B, Smart electric meters, advanced metering infrastructure, and meter communications: Global market analysis and forecasts. In: Navigant research 2014. Boulder, CO, USA; 2014.

[27] European Standard 50160. Voltage characteristics of electricity supplied by public distribution systems. 4th ed.; 2010.

[28] Cigré Task Force C6.04. Benchmark systems for network integration of renewable and distributed energy resources; 2013. ISBN: 978-285-873-270. 